\title{
Millimeter imaging of HD 163296: probing the disk structure and kinematics $\star, \star \star$
}

\author{
A. Isella ${ }^{1,2}$, L. Testi ${ }^{1}$, A. Natta ${ }^{1}$, R. Neri ${ }^{3}$, D. Wilner ${ }^{4}$, and C. Qi ${ }^{4}$ \\ 1 Osservatorio Astrofisico di Arcetri, INAF, Largo E.Fermi 5, 50125 Firenze, Italy \\ e-mail: isella@arcetri.astro.it \\ 2 Dipartimento di Fisica, Universitá di Milano, via Celoria 16, 20133 Milano, Italy \\ 3 Institut de Radio Astronomie Millimétrique (IRAM), 300 rue de la Piscine, Domaine Universitaire de Grenoble, \\ 38406 St. Martin d'Hères, France \\ ${ }^{4}$ Harvard-Smithsonian Center for Astrophysics, 60 Garden Street, MS 42, Cambridge, MA 02138, USA
}

Received 1 March 2007 / Accepted 4 April 2007

\section{ABSTRACT}

\begin{abstract}
We present new multi-wavelength millimeter interferometric observations of the Herbig Ae star HD 163296 obtained with the IRAM/PBI, SMA and VLA arrays both in continuum and in the ${ }^{12} \mathrm{CO},{ }^{13} \mathrm{CO}$ and $\mathrm{C}^{18} \mathrm{O}$ emission lines. Gas and dust properties have been obtained comparing the observations with self-consistent disk models for the dust and $\mathrm{CO}$ emission. The circumstellar disk is resolved both in the continuum and in $\mathrm{CO}$. We find strong evidence that the circumstellar material is in Keplerian rotation around a central star of $2.6 M_{\odot}$. The disk inclination with respect to the line of sight is $46^{\circ} \pm 4^{\circ}$ with a position angle of $128^{\circ} \pm 4^{\circ}$. The slope of the dust opacity measured between 0.87 and $7 \mathrm{~mm}(\beta=1)$ confirms the presence of $\mathrm{mm} / \mathrm{cm}$-size grains in the disk midplane. The dust continuum emission is asymmetric and confined inside a radius of $200 \mathrm{AU}$ while the $\mathrm{CO}$ emission extends up to $540 \mathrm{AU}$. The comparison between dust and $\mathrm{CO}$ temperature indicates that $\mathrm{CO}$ is present only in the disk interior. Finally, we obtain an increasing depletion of $\mathrm{CO}$ isotopomers from ${ }^{12} \mathrm{CO}$ to ${ }^{13} \mathrm{CO}$ and $\mathrm{C}^{18} \mathrm{O}$. We argue that these results support the idea that the disk of HD 163296 is strongly evolved. In particular, we suggest that there is a strong depletion of dust relative to gas outside $200 \mathrm{AU}$; this may be due to the inward migration of large bodies that form in the outer disk or to clearing of a large gap in the dust distribution by a low mass companion.
\end{abstract}

Key words. accretion, accretion disks - techniques: interferometric - stars: planetary systems: protoplanetary disks stars: circumstellar matter

\section{Introduction}

Millimeter and sub-millimeter interferometers are providing an increasingly detailed description of disks around pre-main sequence stars of solar (T Tauri stars; TTS) and intermediate mass (Herbig Ae; HAe). Both dust continuum emission and emission in molecular lines are observed and spatially resolved in a number of disks, yielding information on the disk density and temperature, the dust properties, and the gas chemistry and dynamics in the outer disk (e.g., Natta et al. 2007; Dutrey et al. 2006, and references therein). Thanks to the recent instrumental improvements, it is now possible to build upon the original detections and study more accurately the disk structure details. The number of well-studied disks is however still very small,

^ Based on observations carried out with IRAM Plateau de Bure Interferometer, Submillimeter Array and NRAO Very Large Array. IRAM Plateau de Bure Interferometer is supported by INSU/CNRS (France), MPG (Germany) and IGN (Spain).The Submillimeter Array is a joint project between the Smithsonian Astrophysical Observatory and the Academia Sinica Institute of Astronomy and Astrophysics and is funded by the Smithsonian Institution and the Academia Sinica. The NRAO is a facility of the National Science Foundation operated under cooperative agreement by Associated Universities, Inc.

$\star \star$ Appendix A and Figs. 2-4 are only available in electronic form at http://www. aanda.org practically restricted to the most massive and luminous ones; still, it is clear that disks differ from one another. Recently, evidence of spiral structures and of deviations from Keplerian rotation have been reported in AB Aur, a 2-3 Myr old intermediate mass star, (Piétu et al. 2005; Corder et al. 2005); the classical TTS LkCa15 has a large inner hole of size 50 AU depleted of dust, while the HAe star MWC 480 has a smooth disk with an optically thick (at millimeter wavelengths) inner region of radius 35 AU (Piétu et al. 2006). Both spiral structures and large gaps are evidence of dynamical perturbations, possibly due to the effect of large planets. The existence of both unperturbed and distorted disks among pre-main sequence stars suggests that the planet formation is actively occurring during this evolutionary stage, leaving detectable marks on the parent disks. It is therefore important to study in detail as many disks as possible, in order to characterize their basic properties and to detect deviations from the simple patterns of homogeneous disks in Keplerian rotation.

We report in this paper a detailed study of the disk associated to the HAe star HD 163296, using observations in the continuum and CO lines obtained with three different interferometers, namely the Very Large Array (VLA) at $7 \mathrm{~mm}$, IRAM Plateau de Bure Interferometer (PBI) at 1.3 and $2.6 \mathrm{~mm}$ and the Submillimeter Array (SMA) at $0.87 \mathrm{~mm}$. HD 163296 is a star of spectral type A1, mass of roughly $2.3 M_{\odot}$, distance 
122 pc (van den Ancker et al. 1998). Early OVRO observations (Mannings \& Sargent 1997) have shown the presence of a disk with a minimum mass $\sim 0.03 M_{\odot}$ and evidence of rotation from the $\mathrm{CO}$ lines. The disk is seen in scattered light by Grady et al. (2000, 1999), with radius of $\sim 500 \mathrm{AU}$; it has an associated jet seen in Ly- $\alpha$ with HST, extending on both sides of the disk orthogonally to it (Devine et al. 2000; Wassell et al. 2006). Natta et al. (2004) found evidence of evolved dust in the outer disk of HD 163296 by comparing the VLA $7 \mathrm{~mm}$ flux to the OVRO observations.

The results we present here have much higher spatial resolution and wavelength coverage than what has been previously reported. They allow us to measure accurately the dynamics of the disk as well as the disk and dust properties and to test the capability of disk models to account for the observations. As we will show, they suggest that the HD 163296 system is probably evolving towards a debris disk phase.

The structure of the paper is as follows. Section 2 will describe the observations. The results will be presented in Sect. 3, where we will derive some of the disk parameters. A more detailed analysis, using self-consistent disk models of the dust and CO line emission will be presented in Sect. 4; Sect. 5 contains the results, which will be further discussed in Sect. 6. Summary and conclusions follow in Sects. 7 and 8.

\section{Observations and data reduction}

\subsection{PBI observations}

The PBI observations were carried over the 2003/2004 winter season. The six $15 \mathrm{~m}$ dishes were used in the most extended configuration providing a baseline coverage between 25 and $400 \mathrm{~m}$. The corresponding angular resolutions are reported in Table 1. The receivers were tuned to observe the ${ }^{12} \mathrm{CO} J=2-1$ line and the nearby continuum at $1.3 \mathrm{~mm}$, while at $2.8 \mathrm{~mm}$ the ${ }^{13} \mathrm{CO} J=$ $1-0$, and $\mathrm{C}^{18} \mathrm{O} J=1-0$ lines were observed along with the continuum. Bandpass and complex gain calibrations were ensured by observations of standard IRAM calibrators. The phase stability was excellent during our observations and only a minimal amount of editing of the data was necessary. All calibrations were performed using the standard CLIC suite of programmes within the GILDAS software package. The calibrated uv data were then exported for the subsequent analysis. The accuracy of the flux density scale calibration is expected to be within $20 \%$ at these wavelengths.

\subsection{SMA observations}

The SMA observations of HD 163296 were made on August 23rd, 2005 using the Compact Configuration of seven of the $6 \mathrm{~m}$ diameter antennas, which provided 21 independent baselines ranging in length from 8 to $80 \mathrm{~m}$. The SMA digital correlator was configured with a narrow band of 512 channels over $104 \mathrm{MHz}$, which provided $0.2 \mathrm{MHz}$ frequency resolution, or $0.18 \mathrm{~km} \mathrm{~s}^{-1}$ velocity resolution at $345 \mathrm{GHz}$, and the full correlator bandwidth was $2 \mathrm{GHz}$. The weather was good with $\tau(225 \mathrm{GHz})$ around 0.06 and the double-sideband (DSB) system temperature were between 200 and $500 \mathrm{~K}$. The source HD 163296 was observed from HA -3 to 4.5 . Calibration of the visibility phases and amplitudes was achieved with observations of the quasar 1921-293, typically at intervals of $25 \mathrm{~min}$. Observations of Uranus provided the absolute scale for the flux density calibration and the uncertainties in the flux scale are
Table 1. Columns 2 and 3 show the size and the position angle of the synthesized beam of the HD 163296 observations performed with SMA (at $0.87 \mathrm{~mm}$ ), PBI (at $1.3 \mathrm{~mm}$ and $2.7 \mathrm{~mm}$ ) and VLA (at $7 \mathrm{~mm}$ ). Column 4 show the FWHM size of the detected emission along the major and minor axis of the beam. Column 5 shows the integrated flux with the uncertainties due to the statistical errors.

\begin{tabular}{lllll}
\hline \hline$\lambda$ & Beam & & Emission & $\begin{array}{l}\text { Flux } \\
(\mathrm{mJy})\end{array}$ \\
$(\mathrm{mm})$ & $F W H M$ & PA & $F W H M$ & 20 \\
\hline 0.87 & $3^{\prime \prime} .14 \times 2^{\prime \prime} .21$ & $20^{\circ}$ & $3^{\prime \prime} .60 \times 2^{\prime \prime} .64$ & $1910 \pm 20$ \\
1.3 & $1^{\prime \prime} .95 \times 0^{\prime \prime} .42$ & $7^{\circ}$ & $2^{\prime \prime} .01 \times 0^{\prime \prime} .83$ & $705 \pm 12$ \\
2.8 & $3^{\prime \prime} .3 \times 0^{\prime \prime} .94$ & $8^{\circ}$ & $3^{\prime \prime} .5 \times 1^{\prime \prime} .4$ & $77.0 \pm 2.2$ \\
7 & $1^{\prime \prime} .71 \times 0^{\prime \prime} .81$ & $172^{\circ}$ & $1^{\prime \prime} .71 \times 0^{\prime \prime} .94$ & $4.5 \pm 0.5$ \\
\hline
\end{tabular}

estimated to be $20 \%$. The data were calibrated using the MIR software package ${ }^{1}$.

\subsection{VLA observations}

HD 163296 was observed at the NRAO/VLA as part of a larger survey for $7 \mathrm{~mm}$ disk emission around Herbig Ae stars (see Natta et al. 2004). Data were obtained with the array in the C and D configurations, in several occasions from Dec. 2001 through May 2003. Accurate pointing was ensured by hourly pointing sessions at $3.6 \mathrm{~cm}$ on a bright extra galactic object. The array offered baselines from the shadowing limit through $\sim 3.4 \mathrm{~km}$, although some data was obtained in a hybrid DnA configuration, all the data from the longer baselines had to be rejected due to large phase fluctuations that could not be corrected. The resulting uv-plane coverage offered an angular resolution of $0.5^{\prime \prime}$ and a maximum recoverable size of the order of $\sim 40^{\prime \prime}$. All the data was edited and calibrated using standard recipes in AIPS. The short term complex gain variations were corrected using frequent (few minutes cycle) observations of the quasar 1820-254, while the flux scale was set observing the VLA calibrator $1331+305$. This procedure is expected to be accurate within $\sim 15 \%$ at $7 \mathrm{~mm}$.

\section{Observational results}

The continuum maps obtained at $0.87 \mathrm{~mm}, 1.3 \mathrm{~mm}, 2.8 \mathrm{~mm}$ and $7 \mathrm{~mm}$ are shown in Fig. 1. Channel maps, integrated intensity and mean velocity maps of the ${ }^{12} \mathrm{CO} J=2-1,{ }^{13} \mathrm{CO} J=1-0$ and ${ }^{12} \mathrm{CO} J=3-2$ transitions are shown in Figs. 2-4 respectively. At the wavelength corresponding to the $\mathrm{C}^{18} \mathrm{O} J=1-0$ transition we did not detect any emission; the continuum integrated fluxes are reported in Table 1.

With simple physical assumptions, these observations allow us to determine some fundamental parameters of the star+disk system as the stellar and the disk masses, the contribution to the observed fluxes of free-free gas emission, the wavelength dependence of the observed flux at millimeter wavelengths and the related dust grain opacity.

\subsection{Disk morphology and apparent size}

The $12^{\prime \prime} \times 12^{\prime \prime}$ continuum maps of HD 163296 are shown in Fig. 1. At all wavelengths, the peak is coincident with the position of the optical star as measured from Hipparcos and, given the respective synthesized beams FWHM (see Table 1), the emission is resolved and elongated approximately in the east-west direction. Approximating the source with a circularly symmetric

\footnotetext{
${ }^{1}$ http://cfa-www.harvard. edu/ cqi/mircook.html
} 


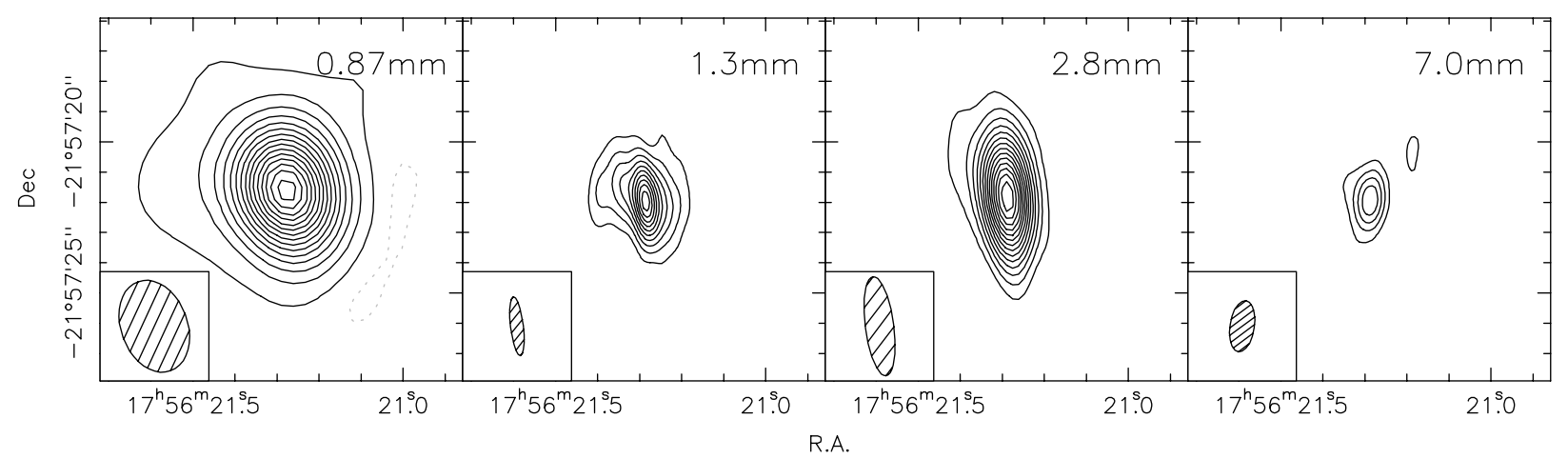

Fig. 1. Continuum maps of HD 163296 at $0.87,1.3,2.8$ and $7 \mathrm{~mm}$, starting from the left. In order to highlight the extended morphology of the disk, the first contour level in the $0.87 \mathrm{~mm}$ map corresponds to $30 \mathrm{mJy}(3 \sigma)$, the second to $6 \sigma$ while the inner contour levels are spaced by $10 \sigma$. At longer wavelength the contour levels are all spaced by $3 \sigma$, corresponding to $12 \mathrm{mJy}$ at $1.3 \mathrm{~mm}, 3.3 \mathrm{mJy}$ at $2.8 \mathrm{~mm}$ and $0.75 \mathrm{mJy}$ at $7 \mathrm{~mm}$. The small boxes show the relative synthesized beams. The integrated fluxes, the beam dimensions and orientations are summarized in Table 1 .

geometrically thin disk and taking into account the beam shape, the observed aspect ratio of the level contours implies an inclination of the disk plane from the line of sight of $45^{\circ} \pm 20^{\circ}$ and a position angle of $120^{\circ} \pm 30^{\circ}$, in rough agreement with the values obtained by Mannings \& Sargent (1997) using marginally resolved OVRO observations ( $58^{\circ}$ and $126^{\circ}$ respectively).

The emission is resolved at all the wavelengths in the EastWest direction. The fainter contour levels are not centrally symmetric, showing excess emission in the east half of the image, better visible in the 0.87 and $1.3 \mathrm{~mm}$ maps. Both the disk size and the morphology will be discussed in more detail in Sect. 6 .

\subsection{Disk kinematics}

As shown in Figs. 2-3, in all the detected molecular line transitions, the emission is resolved, showing a velocity pattern typical of an inclined rotating disk characterized by a position angle of about $130^{\circ}$ (a more precise estimate of the position angle will be presented in Sect. 5). The velocity-position diagram calculated along this direction (see Fig. 5) shows a well defined "butterfly shape" typical of Keplerian rotation. A first estimate of the mass of the central object and of the dimension of the disk can be obtained by comparing the observed velocities with the Keplerian law:

$v_{\epsilon}=C \cdot \epsilon^{-1 / 2}$,

where $\epsilon$ is the angular distance from the central star and $v_{\epsilon}$ is the component of the disk rotational velocity along the line of sight. If the stellar mass $M_{\star}$ is in solar units, the stellar distance $d$ in parsec and $\theta$ is the disk inclination ( $\theta=0$ means pole-on disk), the constant $C \simeq 30 \sqrt{M_{\star} / d} \sin \theta$ is the component along the line of sight of the disk rotational velocity (in $\mathrm{km} \mathrm{s}^{-1}$ ) at $\epsilon=1^{\prime \prime}$. As shown in Fig. 5 the envelopes of both the ${ }^{12} \mathrm{CO}$ and ${ }^{13} \mathrm{CO}$ emissions are in agreement with $C \simeq 2.7 \pm 0.4 \mathrm{~km} \mathrm{~s}^{-1}$. This value corresponds to a stellar mass of $2.0 \pm 0.5 M_{\odot}$ for an inclination of $45^{\circ}$ and $d=122 \mathrm{pc}$. Note finally that the disk rotation is clearly observed at least up to a distance of about $4^{\prime \prime}$, corresponding to a minimum disk outer radius of about $500 \mathrm{AU}$. We will discuss in more detail the determination of the stellar mass and the disk outer radius in Sect. 5, using a detailed model for the continuum and $\mathrm{CO}$ molecular emission.

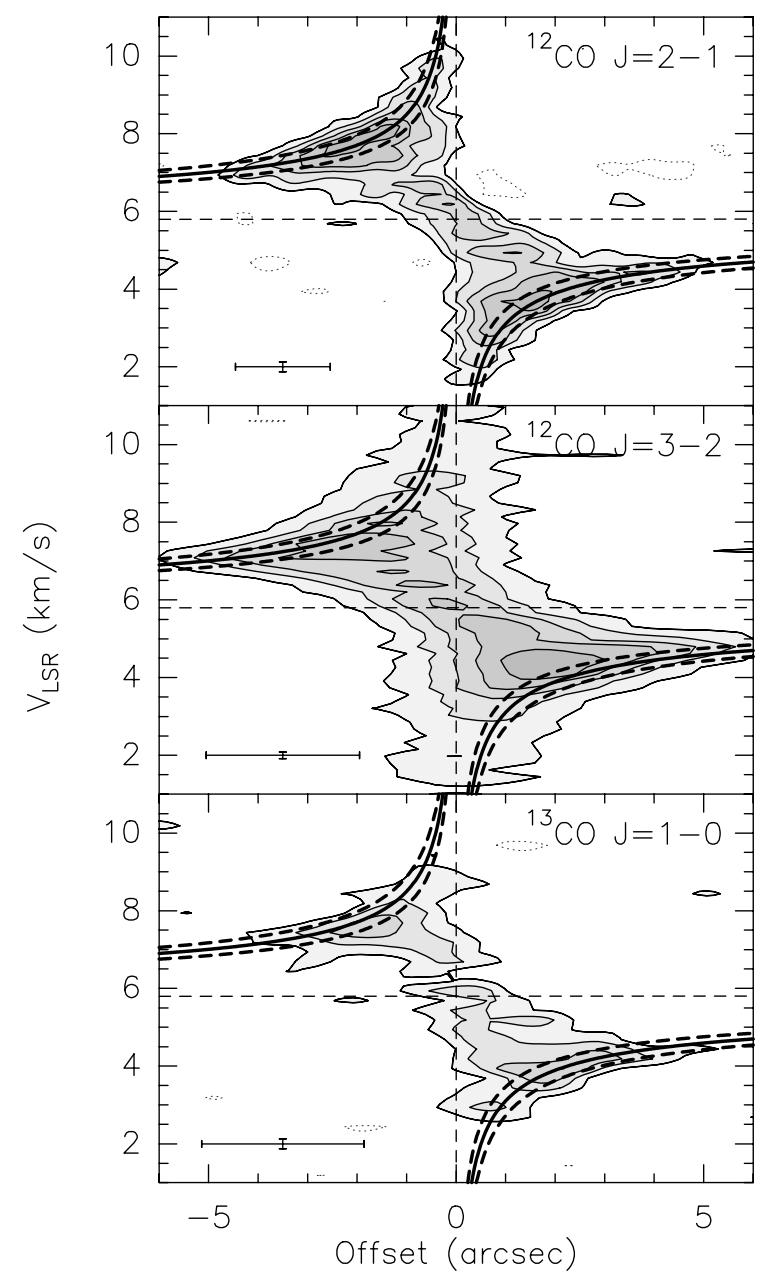

Fig. 5. Velocity-position plots along the plane of the disk for the ${ }^{12} \mathrm{CO} J=2-1$ (upper panel), the ${ }^{12} \mathrm{CO} J=3-2$ (middle panel) and the ${ }^{13} \mathrm{CO} J=1-0$ (lower panel) transitions. The angular offset is measured with respect to the phase center of the observations corresponding to the position of the central star. The contour levels are spaced by $2 \sigma$ corresponding to $0.14 \mathrm{Jy} /$ beam, $1 \mathrm{Jy} /$ beam and $0.06 \mathrm{Jy} /$ beam respectively. The cross in the lower left of each panel gives the angular and spectral resolution of the corresponding map. The thick solid lines marks the border where emission is expected for a Keplerian disk inclined by $45^{\circ}$ and rotating around a $2.0 M_{\odot}$ point source; the external and internal dashed lines correspond to stellar masses of $2.5 M_{\odot}$ and $1.5 M_{\odot}$ respectively. The horizontal and vertical straight dashed lines mark the systemic velocity $\left(5.8 \mathrm{~km} \mathrm{~s}^{-1}\right)$ and the position of the continuum peak. 


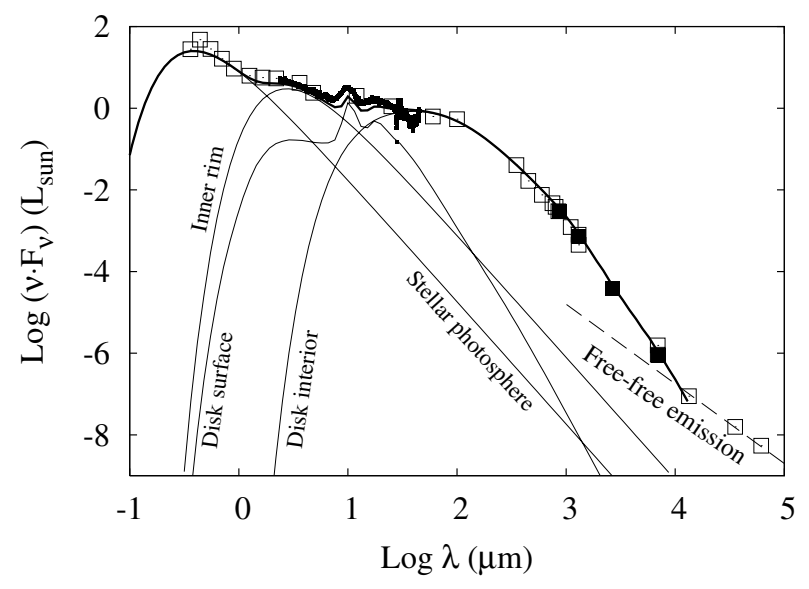

Fig. 6. HD 163296 spectral energy distribution. Data from literature are shown with empty squares (Mannings 1994; Natta et al. 2004; de Winter et al. 2001) and dots (ISO-SW). Our new measurements (see Table 1) are shown with full squares. The observed fluxes at $3.6 \mathrm{~cm}$ and $6.1 \mathrm{~cm}$ have been used to calculate the free-free contribution (dashed line) to the observed flux assuming a free-free spectral index of 0.6. Free-free subtracted fluxes are shown at $7 \mathrm{~mm}$ while at shorter wavelength the free-free contribution is negligible. The solid line shows the prediction of our disk model as discussed in Sect. 5.2. The resulting spectral index $\alpha_{\mathrm{mm}}$ between 0.87 and $7 \mathrm{~mm}\left(F_{\lambda} \propto \lambda^{-\alpha_{\mathrm{mm}}}\right)$ is $3.0 \pm 0.1$.

\subsection{Free-free contribution and spectral index}

The continuum spatially integrated fluxes of HD 163296 and the corresponding spectral energy distribution are given in Table 1 and shown in Fig. 6, respectively. In addition to our measurements (full squares), observations at $0.7,1.3,3.6$ and $6.1 \mathrm{~mm}$ are from Natta et al. (2004, and references therein) while observations at $0.75,0.8,0.85,1.1$ and $1.3 \mathrm{~mm}$ are from Mannings (1994).

Assuming that the observed flux at 3.6 and $6.1 \mathrm{~cm}$ is dominated by free-free emission from a wind (with a spectral index of 0.6), the free-free contribution at $7 \mathrm{~mm}$ corresponds to $1.2 \mathrm{mJy}$ ( $27 \%$ of the observed flux), while it is negligible at shorter wavelengths.

After the subtraction of the free-free component, the millimeter spectral index $\alpha_{\mathrm{mm}}\left(F_{\lambda} \propto \lambda^{-\alpha_{\mathrm{mm}}}\right)$ calculated between $1 \mathrm{~mm}$ and $7 \mathrm{~mm}$ is $3.0 \pm 0.1$, slightly higher than the value $\alpha_{\mathrm{mm}}=2.6 \pm 0.2$ obtained by Natta et al. (2004) using VLA and OVRO fluxes only.

\subsection{Disk mass}

Assuming that the dust emission is optically thin at millimeter wavelengths and that the dust is isothermal, the measured flux can be used to estimate the product of the disk total mass $M$ times the dust opacity $k_{v}$, through the relation:

$M \cdot k_{v}=\frac{F_{v} d^{2}}{B_{v}(T)}$,

where $d$ is the source distance and $T$ is the temperature of the emitting dust. At millimeter wavelengths, the dust opacity can by parametrized by a power law

$k=0.01 \cdot(\lambda / 1.3 \mathrm{~mm})^{-\beta} \mathrm{cm}^{2} \mathrm{~g}^{-1}$,

where the normalization at $\lambda=1.3 \mathrm{~mm}$ assumes a dust/gas mass ratio of 0.01 (Beckwith et al. 1990, 1991). Taking a characteristic temperature for the dust in the outer disk of an early A star of $30 \mathrm{~K}$ (Natta et al. 2000), we derive a total gas+dust mass of $0.12 M_{\odot}$ for the circumstellar material in HD 163296. This rough mass determination, which does not take into account the presence of optically thick emission from the inner part of the disk or of temperature gradients, will be discussed in more detail in Sect. 5.2.

With these simplifying assumptions, the slope of the dust opacity is $\beta=\alpha-2 \sim 1$, where $\alpha$ is the spectral index obtained in the previous section.

\section{Disk models}

The disk parameters derived in Sect. 3 under a number of very crude assumptions can only provide order-of-magnitude estimates. A more quantitative analysis requires comparison of the observations to more sophisticated model predictions. We chose to perform the comparison using the observed visibilities (rather than reconstructed images), following an approach similar to the one discussed by Dutrey et al. (2006).

This method requires to decide "a priori" which family of models is likely to describe the observed object. In view of the results described in Sect. 3, we model the millimeter emission of HD 163296 (continuum and CO lines) as coming from a circumstellar disk. We assume that the disk is heated by the stellar radiation only, and that any viscous contribution can be neglected. This is very likely a good approximation, given the relatively low accretion rate measured in HD $163296\left(\sim 10^{-7} M_{\odot} / \mathrm{yr}\right.$; Garcia Lopez et al. 2006).

The disk structure and emission is computed using the 2-layer approximation of Chiang \& Goldreich (1997), as developed by Dullemond et al. (2001). Similar models have been used in Testi et al. (2003) and Natta et al. (2004), to analyze the (sub)millimeter emission of a number of Herbig Ae stars. We refer to these papers for a more detailed description.

The inner disk is characterized by the presence of a puffed-up inner rim located at the dust evaporation radius (Isella \& Natta 2005). The combined rim+disk flux is calculated taking into account the shadow that the rim casts over the outer disk.

The disk geometry can be fully flared, as in hydrostatic equilibrium disks where gas and dust are well mixed. If dust growth and settling are important, the flaring angle decreases: the resulting radial temperature profile is flatter, affecting the SED in the mid and far infrared (D'Alessio et al. 2006, and references therein). As shown in Fig. 6, the SED of HD 163296 is well reproduced by a fully flared disk model. The fit of the SED becomes very poor if the flaring angle is reduced by more than a factor of two, which corresponds to a small variation of the temperature radial profile.

Once the stellar properties are known, the disk structure is completely characterized by the following parameters: the disk mass $\left(M_{\mathrm{d}}\right)$, the disk outer radius $\left(R_{\text {out }}\right)$, the dependence of the surface density on radius $\left(\Sigma \propto r^{-p}\right)$ and the properties of dust on the disk surface and midplane. In addition, the observed emission depends on the orientation of the disk with respect to the observer, which is characterized by the inclination $\iota$ of the disk with respect to the line of sight ( $\iota=0$ for face-on disks), and the position angle PA.

\subsection{Continuum emission}

The continuum emission at millimeter and sub-millimeter wavelengths is computed by ray integration as in Dullemond et al. (2001). We describe the midplane dust opacity at long wavelengths as a power law of index $\beta$, with $\beta$ a free parameter, as in 
Table 2. Parameters of the disk structure relative to the best fit models for the continuum and the CO emissions as described in Sect. 5. For each parameter uncertainties are given at $1 \sigma$ level.

\begin{tabular}{lllll}
\hline \hline Parameters & Continuum & ${ }^{12} \mathrm{CO} J=2-1$ & ${ }^{12} \mathrm{CO} J=3-2$ & ${ }^{13} \mathrm{CO} J=1-0$ \\
\hline$M_{\star}\left(M_{\odot}\right)$ & $2.6^{a}$ & $2.6_{-0.5}^{+0.3}$ & $2.4 \pm 0.8$ & $2.6 \pm 0.6$ \\
$\mathrm{PA}$ & $120^{\circ} \pm 20^{\circ}$ & $128^{\circ} \pm 5^{\circ}$ & $130^{\circ} \pm 13^{\circ}$ & $130^{\circ} \pm 8^{\circ}$ \\
Incl & $40^{\circ} \pm 12^{\circ}$ & $45^{\circ} \pm 5^{\circ}$ & $45^{\circ} \pm 10^{\circ}$ & $50^{\circ} \pm 8^{\circ}$ \\
$R_{\text {out }}(\mathrm{AU})$ & $200 \pm 15$ & $550 \pm 50$ & $550 \pm 100$ & $500 \pm 80$ \\
$\Sigma_{10 \mathrm{AU}}\left(\mathrm{g} / \mathrm{cm}^{2}\right)$ & $46 \pm 4$ & $90 \pm 70$ & $90^{a}$ & $4_{-3}^{+12}$ \\
$p$ & $0.8 \pm 0.1$ & $0.6_{-0.1}^{+0.3}$ & $0.6^{a}$ & $1.0 \pm 0.5$ \\
$T_{\mathrm{CO}, 100 \mathrm{AU}}(\mathrm{K})$ & & $40_{-5}^{+2}$ & $60 \pm 20$ & $30 \pm 10$ \\
$q$ & & $0.5_{-0.1}^{+0.2}$ & $0.8 \pm 0.4$ & $0.8 \pm 0.4$ \\
$\beta$ & $1.0 \pm 0.1$ & $1.0^{a}$ & $1.0^{a}$ & $1.0^{a}$ \\
$\chi_{r}^{2}$ & 2.6 & 1.17 & 1.05 & 1.15 \\
\hline
\end{tabular}

${ }^{a}$ Fixed parameter.

Eq. (3). At short wavelengths and in the disk surface we adopt the dust opacity of astronomical silicates (Weingartner \& Draine 2001). The inner disk radius is the dust sublimation radius, as in the rim models of Isella et al. (2006) for large $(\sim 1 \mu \mathrm{m})$ grains, and it is equal to $0.45 \mathrm{AU}$; the dust on the disk surface is as in Natta et al. (2004). Neither of these two quantities is relevant for the following analysis.

\subsection{CO emission}

The observed CO emission originates in the outer layers of the disk, at heights that depend on the optical depth of the specific transition. Once the disk structure is specified, as described above, one needs to compute, at each radius, the gas temperature profile in the vertical direction. This is a complex problem, whose results depend on a number of not well known properties, among them the X-ray field and the role of very small grains in heating the gas (e.g., Dullemond et al. 2006). Therefore, we use a parametric description assuming that for each $\mathrm{CO}$ transition the excitation temperature is the same at all $z$ and can be described as a power-law of $r$ in the form:

$T_{\text {line }}=T_{\text {line }}\left(r_{0}\right)\left(r / r_{0}\right)^{-q}$.

The assumption of a constant excitation temperature along the vertical direction is correct for optically thick lines, and/or if the velocity gradient along the line of sight is very large. In both cases, the contribution at each wavelength from the line of sight under consideration comes from a small region only, where the optical depth in the line is of order unity. For any CO line, the height to which $T_{\text {line }}(r)$ refers is therefore different, depending on the density and velocity structure, as well as on the disk inclination angle. The values of $T_{\text {line }}\left(r_{0}\right)$ and $q$ for each CO transition are free parameters. A similar procedure has been used by Dutrey et al. (1994) and Dartois et al. (2003) in their study of the $\mathrm{CO}$ emission of T Tauri disks.

In the analysis of $\mathrm{CO}$ observations we have assumed ${ }^{12} \mathrm{CO} / \mathrm{H}_{2}=7.0 \times 10^{-5},{ }^{13} \mathrm{CO} / \mathrm{H}_{2}=1.0 \times 10^{-6}$ and $\mathrm{C}^{18} \mathrm{O} / \mathrm{H}_{2}=$ $1.3 \times 10^{-7}$, which correspond to the ratios ${ }^{12} \mathrm{CO} /{ }^{13} \mathrm{CO}=70$, ${ }^{12} \mathrm{CO} / \mathrm{C}^{18} \mathrm{O}=550$ (Beckwith \& Sargent 1993; Dutrey et al. 1996, and reference therein)

As described in detail in the Appendix, we have developed a code which computes, for each $\mathrm{CO}$ transition, the line intensity and profile as function of the disk parameters, namely the inclination and PA, the density and temperature profile, and the disk outer radius. In addition, we assume that the disk is in
Keplerian rotation around the central star and vary the stellar mass independently for each line.

In the following, we assume a gas turbulence velocity $v_{\text {turb }}=$ $0 \mathrm{~km} \mathrm{~s}^{-1}$. Note that for $v_{\text {turb }}$ in the range observed in other TTS and HAe (0.07-0.38 $\mathrm{km} \mathrm{s}^{-1}$ from Piétu et al. 2007, 2005, 2003; Dutrey et al. 1994, 1996; Dartois et al. 2003; Simon et al. 2001) the results does not change. This is due to the fact that, for the inclination of the HD 163296 disk, the CO lines width is dominated by the differential disk rotation.

\section{Results}

\subsection{Method of analysis}

The observations of HD 163296 have been analyzed by comparing the observed and the model predicted complex visibilities. For each set of data, the best fit model has been obtained minimizing the $\chi^{2}=\Sigma_{i}\left(\left(\operatorname{Re}_{\mathrm{mod}, i}-\operatorname{Re}_{\mathrm{obs}, i}\right)^{2}+\left(\operatorname{Im}_{\bmod , i}-\operatorname{Im}_{\mathrm{obs}, i}\right)^{2}\right) \cdot W_{i}$, where $\operatorname{Re}_{X}$ and $\operatorname{Im}_{X}$ are the real and the imaginary part of the complex visibility measured (obs) and predicted (mod) in the point $i$ of the uv-plane, and $W_{i}$ is the weight of each measure. For each CO transition, the $\chi^{2}$ has been computed by the simultaneous fitting of 14 velocity channels chosen in order to optimize the sampling of the line profile. The $\chi^{2}$ minimization has been performed exploring a wide region of the space of model parameters. For each parameter, the $1 \sigma$ uncertainties are estimated as $\chi_{1 \sigma}^{2}=\chi_{\mathrm{m}}^{2}+\sqrt{2 n}$, where $n$ is the number of degrees of freedom and $\chi_{\mathrm{m}}^{2}$ is the $\chi^{2}$ value of best fit model.

\subsection{Continuum emission}

The continuum data at the four observed wavelengths $(0.87,1.3$, $2.7,7 \mathrm{~mm}$ ) have been analyzed independently to estimate the best disk parameters and their uncertainties. We have varied the two parameters that define the position of the disk on the plane of the sky, namely the inclination and position angle, as well as the three physical parameters which affect the continuum dust emission, namely the disk outer radius $R_{\text {out }}$, the slope $p$ of the surface density profile and the product $\Sigma_{10 \mathrm{AU}} \times k_{\lambda}$. Since the continuum emission has a very weak dependence on the mass of the central star, we fixed $M_{\star}=2.6 M_{\odot}$ ( see Sect. 5.3).

The values of the parameters obtained at different wavelengths are the same within the uncertainties. In Col. 1 of Table 2 we show the values of the resulting best fit model obtained by combining the independent results at the four different 


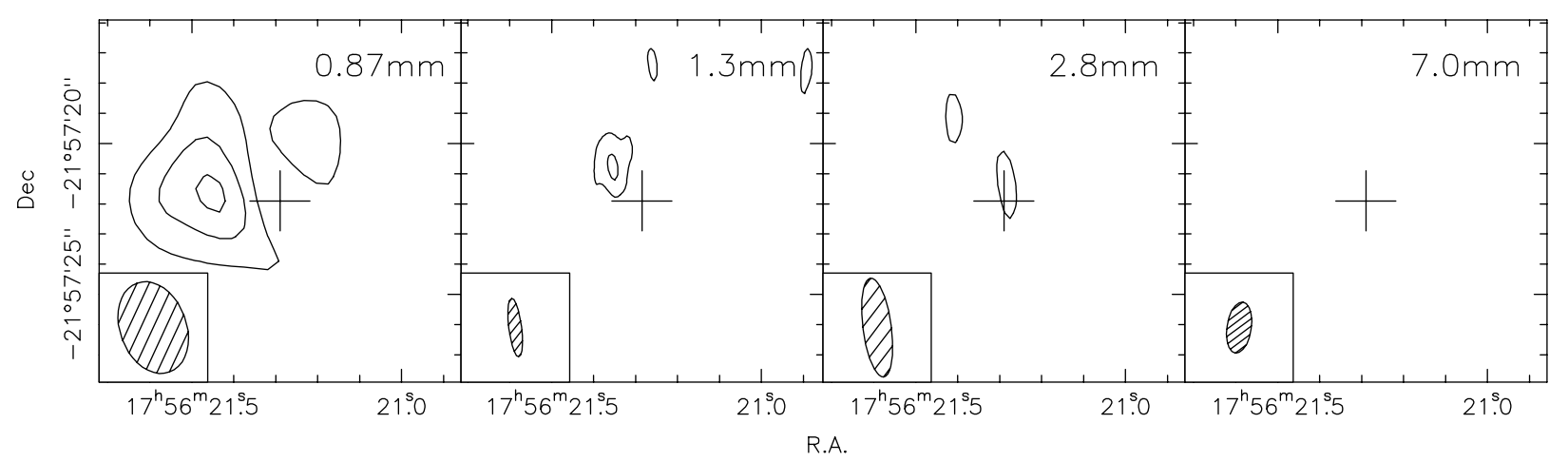

Fig. 7. Maps of the residuals relative to the best fit model for the continuum emission (see Fig. 1 for the observations). The contour level are spaced by $3 \sigma$ corresponding to $30 \mathrm{mJy}$ at $0.87 \mathrm{~mm}, 12 \mathrm{mJy}$ at $1.3 \mathrm{~mm}, 3.3 \mathrm{mJy}$ at $2.8 \mathrm{~mm}$ and $0.75 \mathrm{mJy}$ at $7 \mathrm{~mm}$. The small boxes show the relative synthesized beams.

wavelengths. The constraints are dominated by the $1.3 \mathrm{~mm}$ data, which have the best sensitivity and resolution.

The surface density radial profile has a slope $p=0.81 \pm 0.01$ and the disk outer radius is $R_{\text {out }}=200 \pm 15$ AU. The very good constraints on both parameters are due to the favorable orientation of the beam at $1.3 \mathrm{~mm}$ with the maximum resolution $\left(0.42^{\prime \prime}\right)$ in almost the same direction of the major axis of the disk. Note that the formal error on $p$ is extremely low. On the other hand, small variations on the disk flaring (that allow to fit the SED) lead to correspondingly small variation of the disk radial temperature profile and on the value of $p$. Taking into account this fact, \pm 0.1 is a more reasonable uncertainty for $p$. The formal uncertainties on the other parameters are much larger than those introduced by small variations of the flaring angle.

The four values of $\Sigma_{10 \mathrm{AU}} \times k_{\lambda}$ are used to constrain the slope $\beta$ of the dust opacity law (see Eq. (3)) between 0.87 and $7 \mathrm{~mm}$, which turns out to be $1.0 \pm 0.1$. This confirms the presence of large grains in the HD 163296 circumstellar disk (Natta et al. 2004). Note that the optically thick disk region at $1.3 \mathrm{~mm}$ has size of $\sim 6 \mathrm{AU}$ and contributes only $4 \%$ of the observed flux, so that $\beta$ is very similar to that derived in Sect. 3.3. The observations constrain only the dependence of the dust opacity on wavelength, not its absolute value. The value of $\Sigma_{10} \mathrm{AU}$ given in Table 2 is obtained from $\Sigma_{10 \mathrm{AU}} \times k_{1.3 \mathrm{~mm}}$ assuming $k_{1.3 \mathrm{~mm}}=0.01 \mathrm{~cm}^{2} / \mathrm{g}$.

The residuals for each wavelength are shown in Fig. 7. They have been reconstructed from the residuals in the uv-plane with the same procedure used to obtain the images in Fig. 1. Residual contours are generally lower than $3 \sigma$ with the exception of the 0.87 and $1.3 \mathrm{~mm}$ maps, where a flux asymmetry in the east half of the map (see also Sect. 3.1) is clearly visible. This structure, not detected at longer wavelengths, requires more resolved observations to be investigated in detail.

\subsection{CO emission}

The analysis of the $\mathrm{CO}$ emission has been carried out separately for the different $\mathrm{CO}$ transitions and the corresponding best fit parameters are given in Table 2.

The observed ${ }^{12} \mathrm{CO} J=2-1$ emission is well fitted by a Keplerian disk orbiting a central star with mass $M_{\star}=$ $2.6_{-0.5}^{+0.3} M_{\odot}$. Figure 8 shows the position-velocity residuals obtained subtracting the best fit model from the observed uv-table: no evidence of non-Keplerian rotation or stellar outflow is detected, within the actual instrumental sensitivity. Both the position angle and the inclination are in agreement with the values

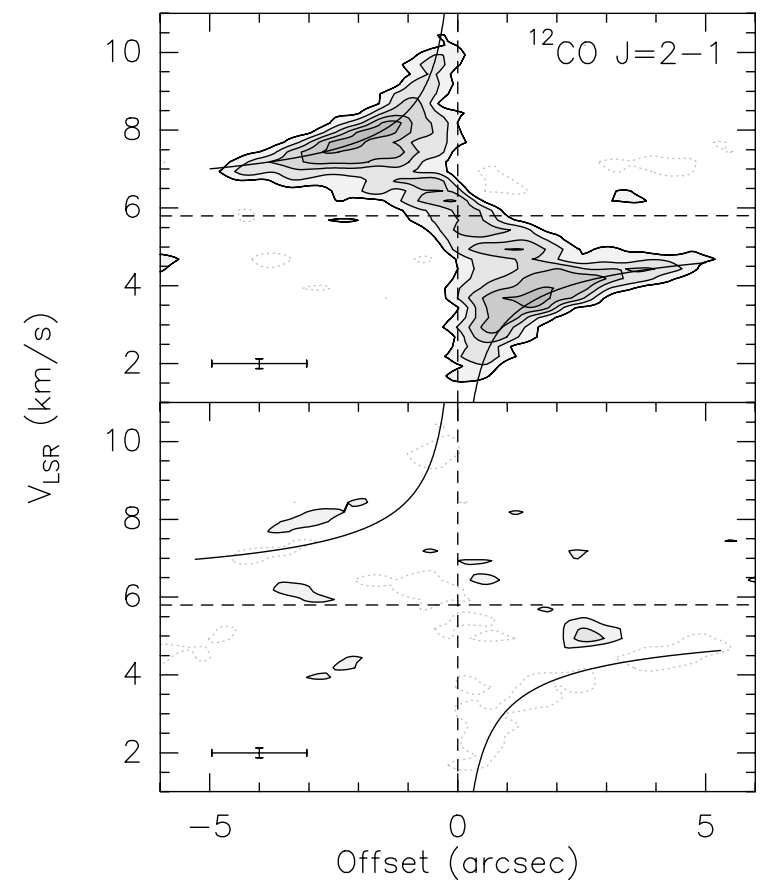

Fig. 8. Comparison between the observed and the model predicted ${ }^{12} \mathrm{CO} J=2-1$ emission. The upper panel shows the position-velocity diagram for the ${ }^{12} \mathrm{CO} J=2-1$ transition (as in Fig. 5). The lower panel shows the residuals relative to the best fit model parameters reported in Table 2. The contour levels are at $2 \sigma$ as in Fig. 5.

obtained from the continuum. Since the line is optically thick, the constraint on the gas surface density is poor. The inferred outer radius of the disk is $550 \pm 50 \mathrm{AU}$, more than two times larger than the value obtained from the continuum and similar to the result obtained by Thi et al. (2004) from the model fitting of single dish line profiles. The radial temperature profile of the ${ }^{12} \mathrm{CO}$ has a slope $q=0.5_{-0.1}^{+0.2}$ and a value of $40_{-5}^{+2} \mathrm{~K}$ at $100 \mathrm{AU}$.

Since the ${ }^{12} \mathrm{CO} J=3-2$ line is optically thick and given the relatively low resolution of the SMA observations, the measurements do not constrain the $\mathrm{CO}$ radial density profile. In this case we fix both $p$ and $\Sigma_{10}$ AU equal to the values obtained for the ${ }^{12} \mathrm{CO} J=2-1$ and vary the other parameters. The results for the inclination, position angle, disk outer radius and $\mathrm{CO}$ temperature are in good agreement with the values obtained for the ${ }^{12} \mathrm{CO} \mathrm{J}=$ $2-1$, with larger uncertainties due to the lower spatial resolution. 
Finally, the model fit to the ${ }^{13} \mathrm{CO} J=1-0$ line gives results consistent with those obtained from the ${ }^{12} \mathrm{CO}$ lines with the exception of the value of $\Sigma_{10}$ AU which is significantly smaller. This discrepancy may be due to a depletion of the ${ }^{13} \mathrm{CO}$ and will be discussed in Sect. 6.5. The radial temperature profile of the ${ }^{13} \mathrm{CO}$ has a slope $q=0.8 \pm 0.4$ and a value of $30 \pm 10 \mathrm{~K}$ at $100 \mathrm{AU}$. We will comment on the gas physical conditions in Sect. 6.5. In general, the parameter constraints obtained from the ${ }^{13} \mathrm{CO}$ are not as good as those obtained for the ${ }^{12} \mathrm{CO} J=2-1$, as expected given the lower resolution of the observations.

\section{Discussion}

\subsection{Disk orientation}

The disk orientation is well determined by our set of measurements: it has a moderate inclination with respect to the line of sight (the mean of the inclination values is $46^{\circ} \pm 4^{\circ}$ ) with a position angle of $128^{\circ} \pm 4^{\circ}$.

\subsection{Disk kinematics and stellar mass}

All the observations are consistent with the emission of a circumstellar disk in Keplerian rotation around a star of $2.6 M_{\odot}$, assuming the Hipparcos distance of $122_{-13}^{+17}$ pc (van den Ancker et al. 1998). Within the error, the stellar mass is in agreement with the value of $2.3 M_{\odot}$ (Natta et al. 2004) obtained from the location of the star on the HR diagram, using Palla \& Stahler (1993) evolutionary tracks; the corresponding stellar age is of about 5 Myr.

\subsection{Disk outer radius}

The model fitting (Table 2) shows that the value of the disk outer radius inferred from the continuum dust emission (200 $\pm 15 \mathrm{AU})$ is almost three times smaller than the value obtained from the $\mathrm{CO}$ analysis (where $540 \pm 40 \mathrm{AU}$ is the mean value). Since our method takes into account the sensitivity limits of the different interferometric observations, this discrepancy can not be explained by the fact that the disk outer regions have a continuum surface brightness below the sensitivity limit. If we extend the disk model that fits the continuum to the outer radius of the $\mathrm{CO}$, we predict a continuum emission between $200 \mathrm{AU}$ and $550 \mathrm{AU}$ which would be easily detected in our observations (see Fig. 9). A similar difference in the dust and gas outer radii has been also reported by Piétu et al. (2005) for the Herbig Ae star AB Aur.

To reconcile the $\mathrm{CO}$ and the dust observations, it is necessary to introduce a sharp drop in the continuum emission of a factor $>30$ at a radius of about 200 AU. With such a drop, the millimeter fluxes at larger $r$ will be below the sensitivity of our observations and will be lost in the observational noise. What can be the origin of such a drop?

In the optically thin regime, which is appropriate for the outer regions of the disk, the continuum flux emitted at distance $r$ from the star depends on the mass surface density $\Sigma(r)$, the dust/gas ratio $\Pi(r)$, the dust opacity $k_{v}(r)$ and the midplane dust temperature $T(r)$ through the relation

$F_{v}(r) \propto \Sigma(r) \cdot \Pi(r) \cdot k_{v}(r) \cdot T(r)$.

It is possible that a different disk geometry (e.g., a lower flaring angle) gives a midplane dust temperature lower than the values predicted by our disk model. A lower temperature limit, of $\sim 10 \mathrm{~K}$, is however imposed by the equilibrium with the interstellar radiation field. Given that our fully flared disk model predicts

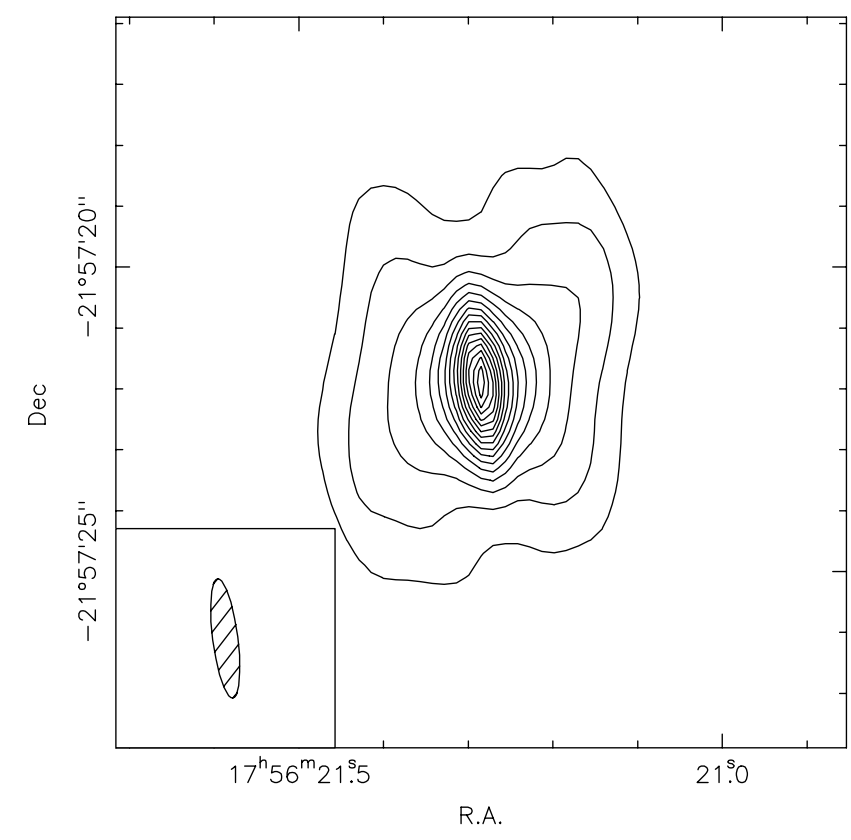

Fig. 9. Model predicted $1.3 \mathrm{~mm}$ continuum emission obtained extrapolating the dust surface density inferred inside the radius of $200 \mathrm{AU}$ to the $\mathrm{CO}$ outer radius of $550 \mathrm{AU}$. The contours are spaced by $3 \sigma$ corresponding to $0.12 \mathrm{mJy}$. See Fig. 1 for the comparison with the observations.

temperatures of $20-30 \mathrm{~K}$ in the millimeter emitting regions, one can reduce $F_{v}$ by a factor 3 at most.

A second possibility to explain the observed flux depletion is that the dust opacity $k_{v}(r)$ at distance larger than $200 \mathrm{AU}$ is much lower because most of the grains have grown into very large bodies.

Finally, a third possibility is that the dust density, i.e. $\Sigma(r)$. $\Pi(r)$, is in some way depleted at large distances. The values of the gas density $\Sigma(r)$ obtained by the CO lines analysis (see Table 2) induce however to exclude the existence of the strong discontinuity in the gas radial density profile required to explain a flux depletion factor $>30$ since a drop of a factor $\sim 10$ in the ${ }^{13} \mathrm{CO}$ distribution at $r>200 \mathrm{AU}$ will already produce small but observable effects on the line emission. On the other hand, it is possible that the ratio dust/gas $\Pi(r)$ decreases very rapidly in the outer disk either due to the formation and fast migration of meter size bodies or to the presence of planetesimals or planets which create a gap in the dust distribution. It is worth noting that the dynamical perturbation induced by such large bodies on the surrounding material (see the review of Papaloizou et al. 2007) may also account for the asymmetric dust density distribution detected in the continuum maps (Fig. 7).

The presence of a giant planet, or a brown dwarf, orbiting in the outer disk of HD 163296 has also be suggested by Grady et al. (2000), in order to explain the dark line observed in the scattered light HST images between 300 AU and 350 AU from the central star. While planets are invoked to explain the large inner gaps observed in the dust distribution in the so called "transitional disks" (i.e., Calvet et al. 2005), HD 163296 will be, if confirmed by future observations, the first case in which a substellar mass companion is found in the outer disk of a pre-main sequence star. 


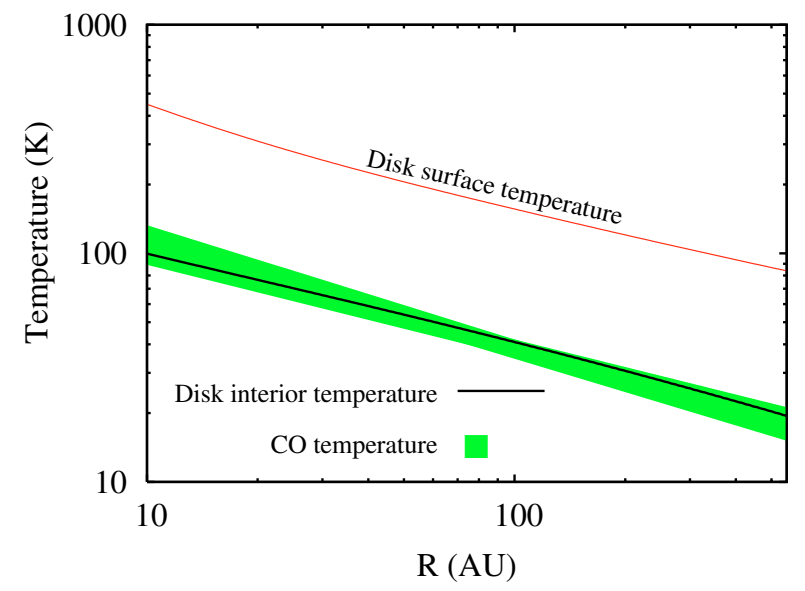

Fig. 10. Comparison between the dust and the CO temperature. As labelled in the figure, the thin-solid line correspond to the dust temperature in the optically thin disk surface, while the thick solid line corresponds to the dust temperature in the disk interior. The CO temperature is indicated with the shaded region, taking into consideration the uncertainties. The model parameters are those reported in Col. 3 of Table 2.

\subsection{Disk surface density and mass}

Both the dust continuum emission and the $\mathrm{CO}$ lines indicate a rather shallow surface density profile, $\Sigma \propto r^{-0.8}$ corresponding to a gas + dust mass of $0.05 \pm 0.01 M_{\odot}$ if the disk outer radius is $200 \mathrm{AU}$. However, if, as we believe, the gaseous disk extends with the same surface density profile all way to $540 \mathrm{AU}$ the inferred disk mass is much larger, $0.17 M_{\odot}$.

An additional uncertainty on the disk mass comes from the uncertainty on the dust opacity, as discussed in Natta et al. (2007). Although a disk mass of $0.17 M_{\odot}\left(6 \%\right.$ of $\left.M_{\star}\right)$ is probably a lower limit, the observed Keplerian rotation pattern indicates that the disk should not be much more massive. In this case one expects deviations from Keplerian rotation (Lodato \& Bertin 2003) as observed in massive disks (Cesaroni et al. 2005).

The example of HD 163296 illustrates how the simple disk mass derivation from the observed millimeter flux (see Sect. 3.4) can be wrong for a variety of reasons, even when the emission is optically thin. In the case of HD 163296, only $30 \%$ of the total mass (i.e., the fraction inside $200 \mathrm{AU}$ ) contributes to the continuum millimeter flux. On the other hand, the disk is hotter than the $30 \mathrm{~K}$ assumed in Eq. (2). The two effects cancel in part so that the "simple" and "correct" values differ by about 50\% (for the same $k_{1.3 \mathrm{~mm}}$ normalization).

\subsection{CO vs. dust temperature}

At the actual gas density both the ${ }^{12} \mathrm{CO}$ and ${ }^{13} \mathrm{CO}$ lines are optically thick and lead to similar $\mathrm{CO}$ excitation temperatures expressed by the relation $T(r) \sim 40 \mathrm{~K} \cdot(r / 100 \mathrm{AU})^{-0.5}$. Figure 10 shows the comparison between this CO temperature (shaded region) and the dust temperature corresponding to the same disk model. The disk interior temperature $T_{i}$ (thick-solid line) and the disk surface temperature $T_{\mathrm{s}}$ (thin-solid line), calculated using our two-layer disk model, correspond respectively to disk regions where $\tau_{\star} \gg 3$ and $\tau_{\star} \simeq 1$ (Dullemond et al. 2002); $\tau_{\star}$ is the optical depth for the stellar radiation calculated along the radial direction. The equivalence between the $\mathrm{CO}$ and the dust temperature can thus be interpreted as the evidence that the $\mathrm{CO}$, and in particular the ${ }^{12} \mathrm{CO}$, lines are emitted by the gas present at such values of the optical depth, e.g. well under the disk



Fig. 11. Ratio between the Planck mean opacity at the stellar temperature $k_{\star}^{P}$ and the UV opacity $k_{\mathrm{UV}}$ for different grain sizes and composition. The upper panel shows the opacity ratio for compact silicate grains; the lower panel shows the ratio for porous grains composed by Olivine (17\%), carbonates (53\%) and vacuum (30\%). The solid lines are relative to an HAe star of $T_{\star}=10000 \mathrm{~K}$, the long-dashed lines refer to a TTS with $T_{\star}=4000 \mathrm{~K}$ and the short-dashed lines to the same TTS with the addition of the UV excess radiation calculated so that $L_{\mathrm{UV}} / L_{\star}=0.05$. This latter case is representative for the emission released by the gas accreting on the stellar surface.

surface. Is this result compatible with the high optical depth of the ${ }^{12} \mathrm{CO}$ lines?

Assuming that $\mathrm{H}_{2}$ and $\mathrm{CO}$ are well mixed all over the vertical extension of the disk and assuming also the standard ratio ${ }^{12} \mathrm{CO} / \mathrm{H}_{2}=7.0 \times 10^{-5}$, the ${ }^{12} \mathrm{CO}$ lines should be emitted by disk regions well above the disk surface, defined as the height where $\tau_{\star}=1$. In this situation, we expect a CO temperature close to the disk surface temperature, or even higher if the gas and the dust are decoupled (Jonkheid et al. 2007). On the other hand CO molecules on the superficial layers of the disk are expected to be photo-dissociated by the UV photons emitted by the hot central star. In particular, for an Herbig Ae star similar to HD 163296, Jonhkeid et al. (2007) show that the CO photodissociation occurs in the disk regions where the optical depth to the stellar UV radiation field is $\tau_{\mathrm{UV}} \lesssim 3$ (see Fig. 5.6 therein). Therefore, the $\mathrm{CO}$ and disk interior temperature are expected to be similar if $\tau_{\mathrm{UV}} \lesssim \tau_{\star}$, i.e., if $k_{\mathrm{UV}} \lesssim k_{\star}^{P}$, where $k_{\star}^{P}$ is the dust opacity averaged on the stellar spectrum. We think that this is the case in HD 163296.

The dust opacity is a complex quantity that depends on the dust chemical composition, structure and on the grain size distribution. The condition $k_{\mathrm{UV}} \lesssim k_{\star}^{P}$ is generally satisfied by large grains. In the case of HD 163296 one needs, for example, compact silicate grains larger than $0.1 \mu \mathrm{m}$ or porous grains of silicate and carbonaceous materials larger than few microns (see Fig. 11). Cooler stars require larger grains, unless significant UV excess is present.

If $\mathrm{CO}$ survives only in the disk interior, we expect that lines with different optical depth will have similar temperatures. On the contrary, if $\mathrm{CO}$ is present in the disk surface layers, lines with higher optical depth should have higher excitation temperatures.

The few existing observations indicate that all cases occur. Piétu et al. (2007) have recently reported that for the HAe stars MWC 480 and AB Aur and the TTS DM Tau, the ${ }^{12} \mathrm{CO}$ and ${ }^{13} \mathrm{CO}$ lines are characterized by different temperatures, while in the case of the TTS LkCa 15 the CO temperatures are all similar. We suggest that these differences are due to different dust 
properties (i.e. different composition, grain growth and settling) and stellar spectra.

\subsection{CO isotopic abundances}

The values of $\Sigma_{10}$ AU reported in Table 2 indicate that the ${ }^{13} \mathrm{CO} J=1-0$ emission requires a ratio ${ }^{13} \mathrm{CO} / \mathrm{H}_{2} \sim 10^{-7}$, about a factor 10 lower than what found in interstellar clouds. From the non-detection of the $\mathrm{C}^{18} \mathrm{O} J=1-0$ line we estimate that the $\mathrm{C}^{18} \mathrm{O}$ should be depleted of a factor $>60$ with respect to the typical ratio ${ }^{12} \mathrm{CO} / \mathrm{C}^{18} \mathrm{O}=550$. We note that a similar trend of depletion from ${ }^{12} \mathrm{CO}$ to ${ }^{13} \mathrm{CO}$ and $\mathrm{C}^{18} \mathrm{O}$ has been found by Dutrey et al. $(1994,1996)$ in a number of TTS. On the other hand an overabundance of ${ }^{13} \mathrm{CO}$ has been found in DM Tau, MWC 480 and LkCa 15 (Piétu et al. 2007) while for DM Tau, Dartois et al. (2003) find that all the $\mathrm{CO}$ isotopomers $\left({ }^{12} \mathrm{CO},{ }^{13} \mathrm{CO}\right.$ and $\left.\mathrm{C}^{18} \mathrm{O}\right)$ are depleted by the same factor $\sim 10$. The observational picture is still very uncertain and deserves further investigations.

In the case of HD 163296, the gas temperature always higher than $20 \mathrm{~K}$ rules out the condensation of $\mathrm{CO}$ onto dust grains as possible cause of the $\mathrm{CO}$ isotopomers depletion. Alternatively, Jonkheid et al. (2007) have recently pointed out that a higher ratio between the ${ }^{12} \mathrm{CO}$ and its isotopomers may be the consequence of grain growth and settling. This latter hypothesis is more convincing given the evidence of grain growth in the HD 163296 disk discussed in this work (see Sects. 5.2 and 6.5).

\section{Summary}

This paper presents new observations of the disk of HD 163296 in the dust continuum from 0.87 to $7 \mathrm{~mm},{ }^{12} \mathrm{CO}(J=2-1$ and $J=3-2)$ and ${ }^{13} \mathrm{CO}(J=1-0)$ lines. The disk is resolved in all lines and continuum.

We have compared the observations to the predictions of self-consistent disk models. We find that the disk, as seen in CO lines, is very large $(R=540 \mathrm{AU})$, with a Keplerian rotation pattern consistent with a central mass of $2.6 M_{\odot}$. Within the observational errors, there is no evidence of non-Keplerian motions and/or significant turbulent broadening. We obtain a disk inclination of $46^{\circ}$, significantly lower than the value of $\sim 60^{\circ}$ found in literature.

The dust opacity has a power law dependence on wavelength $\kappa \propto \lambda^{-\beta}$ with $\beta=1.0 \pm 0.1$ in the interval $0.87-7 \mathrm{~mm}$. This value is similar to what has been measured in a number of spatially resolved disks of HAe and TTS (e.g., Natta et al. 2004; Rodmann et al. 2006), and is very likely an indication that the bulk of the solid material in these disks has coagulated into very large bodies, of millimeter and centimeter size (Natta et al. 2007). Within the accuracy of our data we do not confirm the possible variation of $\beta$ with $r$ discussed in Natta et al. (2007).

The continuum observations constrain the surface density profile $\left(\Sigma \propto r^{0.8}\right)$ for $r \leq 200$ AU. At larger radii, the continuum emission drops with respect to the model predictions by a factor 30 at least (at $1.3 \mathrm{~mm}$ ). We argue that this may be due to the clearing of a very large gap by dynamical perturbations from a low mass companion or to the inward migration of the large bodies that may form in the outer disk.

The temperatures derived for CO lines of different optical depth are similar and equal to the dust temperature in the disk interior $\left(\tau_{\star} \gg 1\right)$. This requires that the dust opacity in the $\mathrm{UV}$ (which controls the $\mathrm{CO}$ dissociation) and in the wavelength range where the stellar radiation peaks are similar, as expected if grains have grown to micron size. Differences in the temperature of the $\mathrm{CO}$ lines can only be expected if $\mathrm{CO}$ is present in the disk surface layers. A variety of situations can occur depending on the exact dust composition and sizes and on the stellar radiation field.

A comparison of the disk properties derived from the dust continuum and the $\mathrm{CO}$ lines shows that the ${ }^{13} \mathrm{CO} J=1-0$ emission is consistent with a ratio of ${ }^{13} \mathrm{CO} / \mathrm{H}_{2}$ and $\mathrm{C}^{18} \mathrm{O} / \mathrm{H}_{2}$ about a factor 10 and 60 lower than what is found in interstellar clouds.

\section{Conclusions}

The results discussed in this work can be interpreted as clues to the evolution occurring in the HD 163296 system. The presence of large grains in the disk midplane, the equivalence between the dust and CO temperature, the drop in the continuum dust emission further out $200 \mathrm{AU}$, its detected asymmetry and, maybe, the $\mathrm{CO}$ isotopomers depletion, all support the idea that the circumstellar disk is probably harboring the formation of large bodies, being in between a Class II pre-main sequence disk and an older debris disk.

On the other hand, it is important to underline that HD 163296 strongly differs from the so called transitional disks (Calvet et al. 2005) characterized by a clearing of the inner disk supposed to be originated by the presence of a giant planet. In the case of HD 163296, it is the outer disk that appear dust depleted. This conclusion opens a number of theoretical problem about how dust grains can growth in the low density outer disk environment. In this respect the recently improved PBI array, the new CARMA array and ALMA will bring in the next future to real observational breakthrough in the comprehension of circumstellar disk evolution and planetary formation.

Acknowledgements. The authors acknowledge partial support for this project by MIUR PRIN grant 2003/027003-001. A.I. acknowledge Riccardo Cesaroni, Malcolm Walmsley, Giuseppe Bertin, Ewine van Dishoek and Anneila Sargent for their help and useful suggestions.

\section{References}

Beckwith, S. V. W., \& Sargent, A. I. 1991, ApJ, 381, 250

Beckwith, S. V. W., \& Sargent, A. I. 1993, ApJ, 402, 280

Beckwith, S. V. W., Sargent, A. I., Chini, R. S., \& Guesten, R. 1990, AJ, 99, 924

Calvet, N., D’Alessio, P., Watson, D. M., et al. 2005, ApJ, 630, 185

Cesaroni, R., Galli, D., Lodato, G., Walmsley, C. M., \& Zhang, Q. 2006, in Protostars and Planets V, ed. B. Reipurth, D. Jewitt, \& K. Keil (Tucson: University of Arizona Press), in press

Chiang, E. I., \& Goldreich, P. 1997, ApJ, 490, 368

Corder, S., Eisner, J., \& Sargent, A. I. 2005, ApJ, 622L, 133C

D’Alessio, P., Calvet, N., \& Hartmann, L. 2001, ApJ, 554, 321

D’Alessio, P., Calvet, N., Hartmann, L., Franco-Hernández, R., \& Servìn, H. 2006, ApJ, 638, 314

Dartois, E., Dutrey, A., \& Guilloteau, S. 2003, A\&A, 399, 773

Devine, D., Grady, C. A., Kimble, R. A., et al. 2000, ApJ, 542, 115

de Winter, D., van den Ancker, M. E., Maira, A., et al. 2001, A\&A, 380, 609

Dominik, C., Blum, J., Cuzzi, J. N., \& Wurm, G. 2007, in Protostars and Planets V, ed. B. Reipurth, D. Jewitt, \& K. Keil (Tucson: University of Arizona Press), 783

Dullemond, C. P., Dominik, C., \& Natta, A. 2001, ApJ, 560, 957

Dullemond, C. P., van Zadelhoff, G. J., \& Natta, A. 2002, A\&A, 389, 464

Dullemond, C. P., Hollenbach, D., Kamp, I., \& D’Alessio, P. 2006, in Protostars and Planets V, ed. B. Reipurth, D. Jewitt, \& K. Keil (Tucson: University of Arizona Press), in press

Dutrey, A., Duvert, G., Castets, A., et al. 1993, A\&A, 270, 468

Dutrey, A., Guilloteau, S., \& Simon, M. 1994, A\&A, 286, 149

Dutrey, A., Guilloteau, S., Duvert, G., et al. 1996, A\&A, 309, 493

Dutrey, A., Guilloteau, S., Prato, L., et al. 1998, A\&A, 338, L63

Dutrey, A., Guilloteau, S., \& Ho, P. 2007, in Protostars and Planets V, ed. B. Reipurth, D. Jewitt, \& K. Keil (Tucson: University of Arizona Press), 495 
Garcia Lopez, R., Natta, A., Testi, L., \& Habart, E. 2006, A\&A, 459, 837 Grady, C. A. 2000, ApJ, 544, 859

Grady, C. A., Woodgate, B., Bruhweiler, F. C., et al. 1999, ApJ, 523, L151

Jonkheid, B., Dullemond, C. P., Hogerheijde, M. R., \& van Dishoeck, E. F. 2007, A\&A, 463, 203

Isella, A., \& Natta, A. 2005, A\&A, 438, 899

Isella, A., Testi, L., \& Natta, A. 2006, A\&A, 451, 951

Lodato, G., \& Bertin, G. 2003, A\&A, 408, 1015

Mannings, V. 1994, MNRAS, 271, 587

Mannings, V., \& Sargent, A. I. 1997, ApJ, 490, 792

Natta, A., Grinin, V. P., \& Mannings, V. 2000, in Protostars and Planets IV, ed. V. Mannings, A. P. Boss, \& S. S. Russell (Tucson: Univ. of Arizona Press), 559 Natta, A., Testi, L., Neri, R., Shepherd, D. S., \& Wilner, D. J. 2004, A\&A, 416, 179

Natta, A., Testi, L., Calvet, N., et al. 2007, in Protostars and Planets V, ed. B. Reipurth, D. Jewitt, \& K. Keil (Tucson: University of Arizona Press), 767

Papaloizou, J. C. B., Nelson, R. P., Kley, W., Masset, F. S., \& Artymowicz, P. 2007, in Protostars and Planets V, ed. B. Reipurth, D. Jewitt, \& K. Keil (Tucson: University of Arizona Press), 655
Palla, F., \& Sthaler, S. W. 1993, ApJ, 418, 414

Piétu, V., Dutrey, A., \& Kahane, C. 2003, A\&A, 398, 565

Piétu, V., Guilloteau, S., \& Dutrey, A. 2005, A\&A, 443, 945

Piétu, V., Guilloteau, S., Dutrey, A., Chapillon, E., \& Pety, J. 2006, A\&A, 460, L43

Piétu, V., Dutrey, A., \& Guilloteau, S. 2007, A\&A, in press [arXiv: astro-ph/0701425]

Rodmann, J., Henning, Th., Chandler, C. J., Mundy, L. G., \& Wilner, D. J. 2006, A\&A, 446, 211

Semenov, D., Henning, Th., Helling, Ch., Ilgner, M., \& Sedlmayr, E. 2003, A\&A, 410, 611

Simon, M., Dutrey, A., \& Guilloteau, S. 2001, ApJ, 545, 1034

Testi, L., Natta, A., Shepherd, D. S., \& Wilner, D. J. 2003, A\&A, 403, 323

Thi, W.-F., van Zedelhoff, G.-J., \& van Dishoeck, E. F. 2004, A\&A, 425, 955

Wassell, E. J., Grady, C. A., Woodgate, B., Kimble, R. A., \& Bruhweiler, F. C. 2006, ApJ, 650, 985

Weingartner, J. C., \& Draine, B. T. 2001, ApJ, 548, 296

van den Ancker, M. E., de Winter, D., \& Tjin A Djie, H. R. E. 1998, A\&A, 330, $145 \mathrm{~V}$ 
A. Isella et al.: Millimeter imaging of HD 163296, Online Material p 1

\section{Online Material}


A. Isella et al.: Millimeter imaging of HD 163296, Online Material p 2

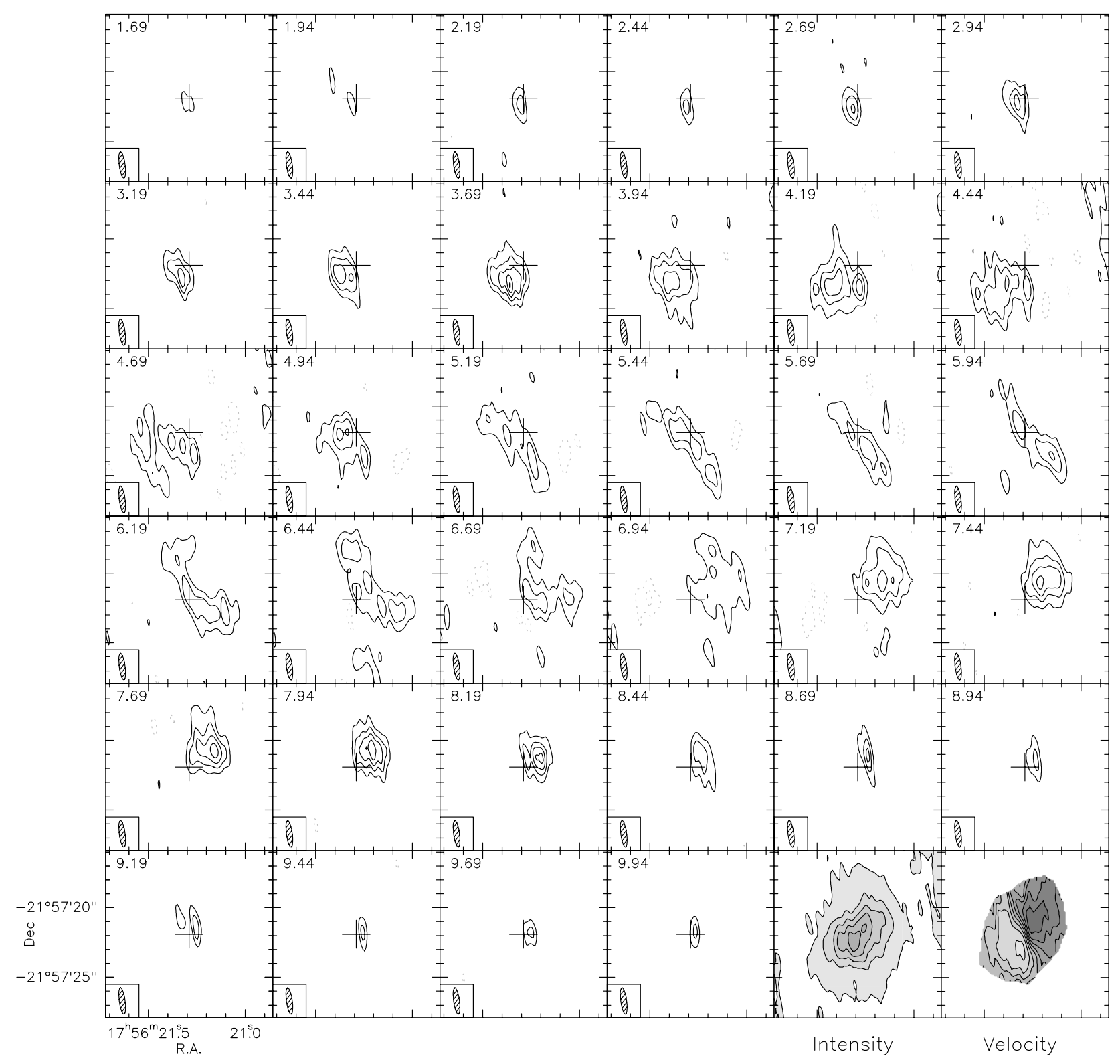

Fig. 2. Velocity channel maps of the ${ }^{12} \mathrm{CO} J=2-1$ emission. The velocity resolution is $0.25 \mathrm{~km} \mathrm{~s}^{-1}$. The LSR velocity is indicated in the upper left corner of each panel. The angular resolution (synthesized beam), indicated in the small boxes, is $1.95^{\prime \prime} \times 0.42^{\prime \prime}$ at PA $7^{\circ}$; the contour spacing is $0.23 \mathrm{Jy} /$ beam corresponding to $3 \sigma$. The last two panels show the integrated intensity (contour levels spaced by $0.4 \mathrm{Jy} / \mathrm{beam}^{\mathrm{km} \mathrm{s}}{ }^{-1}$ ) and the velocity field (contour levels from $3 \mathrm{~km} \mathrm{~s}^{-1}$ to $9 \mathrm{~km} \mathrm{~s}^{-1}$ spaced by $0.5 \mathrm{~km} \mathrm{~s}^{-1}$ ) respectively. 
A. Isella et al.: Millimeter imaging of HD 163296, Online Material p 3

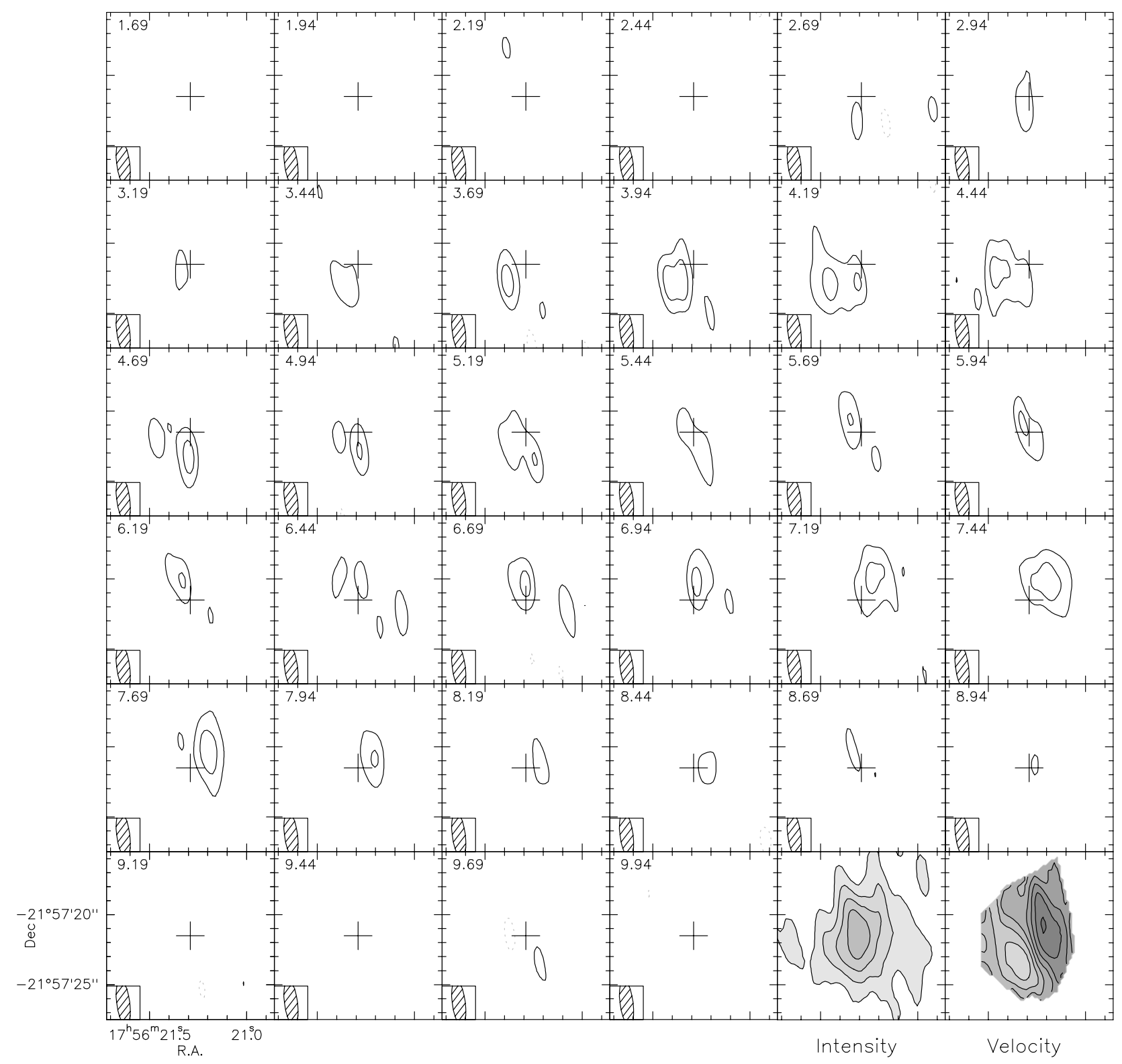

Fig. 3. Velocity channel maps of the ${ }^{13} \mathrm{CO} J=1-0$ emission. The velocity resolution is $0.25 \mathrm{~km} \mathrm{~s}^{-1}$. The LSR velocity $\left(\mathrm{km} \mathrm{s}^{-1}\right)$ is indicated in the upper left corner of each panel. The angular resolution (synthesized beam), indicated in the small boxes, is $3.3^{\prime \prime} \times 0.94^{\prime \prime}$ at PA $8^{\circ}$; the contour spacing is $0.09 \mathrm{Jy} /$ beam corresponding to $3 \sigma$. The last two panels show the integrated intensity (contour levels spaced by $0.12 \mathrm{Jy} / \mathrm{beam}$ ) and the velocity field (contour levels from $3 \mathrm{~km} \mathrm{~s}^{-1}$ to $9 \mathrm{~km} \mathrm{~s}^{-1}$ spaced by $0.5 \mathrm{~km} \mathrm{~s}^{-1}$ ) respectively. 
A. Isella et al.: Millimeter imaging of HD 163296, Online Material p 4

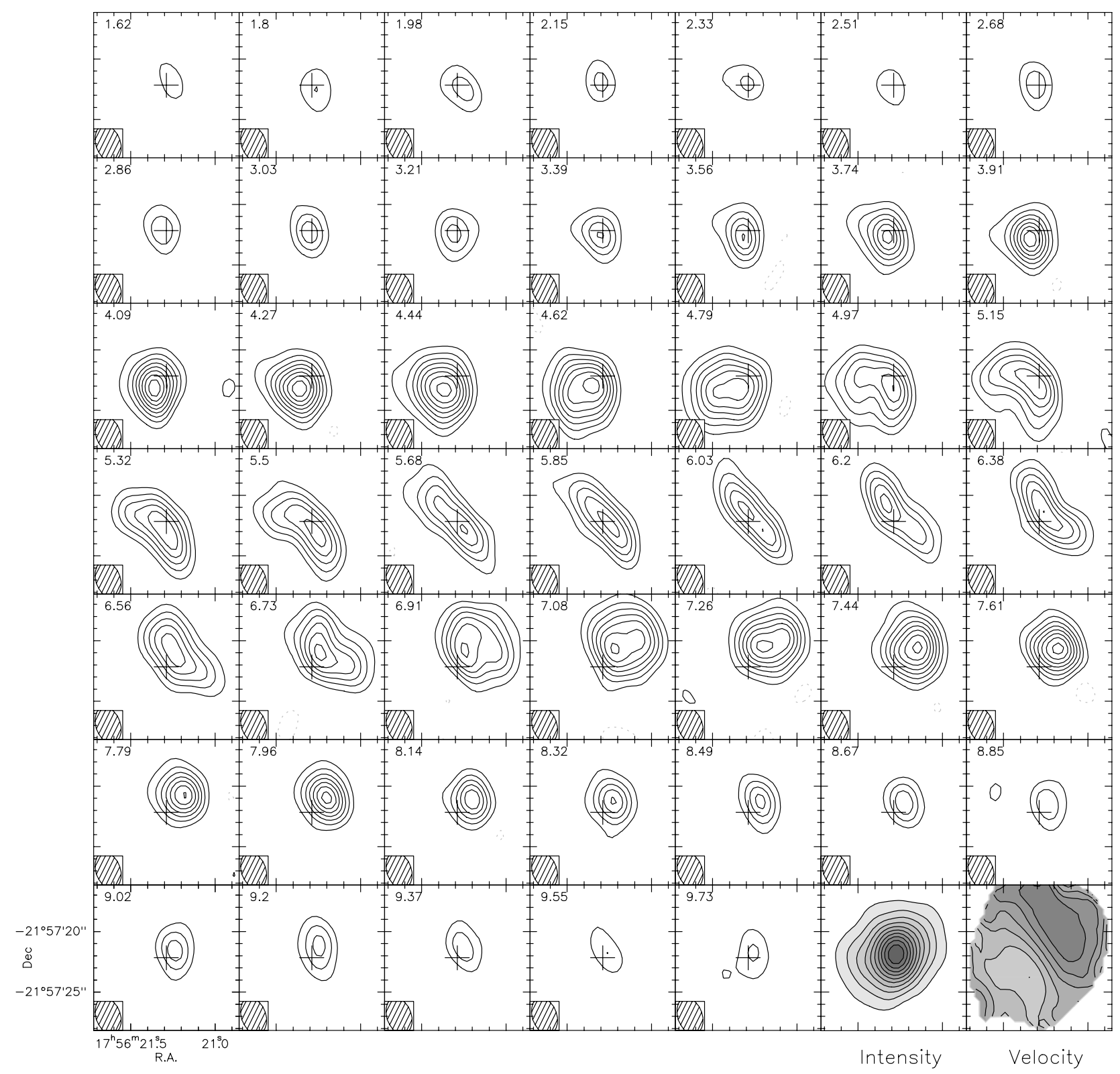

Fig. 4. Velocity channel maps of the ${ }^{12} \mathrm{CO} J=3-2$ emission. The velocity resolution is $0.18 \mathrm{~km} \mathrm{~s}^{-1}$. The LSR velocity $\left(\mathrm{km} \mathrm{s}^{-1}\right)$ is indicated in the upper left corner of each panel. The angular resolution (synthesized beam), indicated in the small boxes, is $3.4^{\prime \prime} \times 2.21^{\prime \prime}$ at PA $20^{\circ}$; the contour spacing is $1.5 \mathrm{Jy} / \mathrm{beam}$ corresponding to $3 \sigma$. The last two panels show the integrated intensity (contour levels spaced by $5 \mathrm{Jy} / \mathrm{beam} \mathrm{km} \mathrm{s}^{-1}$ ) and the velocity field (contour levels from $3 \mathrm{~km} \mathrm{~s}^{-1}$ to $9 \mathrm{~km} \mathrm{~s}^{-1}$ spaced by $0.5 \mathrm{~km} \mathrm{~s}^{-1}$ ) respectively. 


\section{Appendix A: CO emission model}

In order to analyze the $\mathrm{CO}$ emission we developed a numerical code that solve the general formulation of the radiation transfer equation along each direction between the observer and the emitting source. If $s$ is the linear coordinate along the line of sight, increasing from the observer $(s \equiv 0)$ towards the source, the observed emission in each direction is given by the relation

$I_{v}=\int_{0}^{\infty} S_{v}(s) \mathrm{e}^{-\tau_{v}}(s) K_{v}(s) \mathrm{d} s$,

where the optical depth is defined in each point $s$ through

$\tau_{v}(s)=\int_{0}^{s} K_{v}\left(s^{\prime}\right) \mathrm{d} s^{\prime}$

and $K_{v}$ is the absorbing coefficient of the interstellar medium. Given the high gas densities in the pre-main sequence circumstellar disks, we can assume that all the CO levels corresponding to the rotational transitions under investigation are thermalized. In this case the source function can be approximated by the Planck function

$S_{v}(s)=B_{v}\left(T_{\mathrm{CO}}(s)\right)=\frac{2 h v^{3}}{c^{2}} \frac{1}{\exp \left(h v / k T_{\mathrm{CO}}(s)\right)-1}$,

depending only on the local temperature of the gas $T_{\mathrm{CO}}(c$, $h$ and $k$ are respectively the light speed, the Planck and Boltzman constant).

The absorbing coefficient of the circumstellar medium is due both to gaseous CO and dust: $K_{v}(s)=K_{v}^{\mathrm{CO}}(s)+K_{v}^{d}(s)$. For the dust $K_{v}^{d}(s)=\rho(s) \cdot k_{v}$, where $\rho(s)$ is the local density of the circumstellar material (gas+dust) and $k_{v}$ is the dust absorbing coefficient for total mass unit given in Eq. (3). The CO absorbing coefficient is given by the relation

$K_{v}^{\mathrm{CO}}(s)=n_{l}(s) \cdot \sigma_{v}(s)$

where $n_{l}(s)$ is the total number of CO molecules at the lower level $l$ of the transition and $\sigma_{v}(s)$ is the CO absorbing cross section.

Calling $m_{0}$ and $\chi_{\mathrm{CO}}$ the mean molecular weight of the gas and the fraction of $\mathrm{CO}$ present in the gas respectively, the number of molecules $n_{l}(s)$ is given by the Boltzman equation

$n_{l}(s)=\chi_{\mathrm{CO}} \frac{\rho(s)}{m_{0}} \cdot \frac{g_{l} \mathrm{e}^{-E_{l} / k T_{\mathrm{CO}}(s)}}{Z\left(T_{\mathrm{CO}}(s)\right)}$,

where $g_{l}=2 l+1$ is the statistical weight of the lower level $l$ of the transition, $E_{l}=(1 / 2) l(l+1) k T_{1}$ is the level energy, $T_{1}$ is the temperature equivalent to the transition energy and $Z\left(T_{\mathrm{CO}}(s)\right)$ is the partition function at the gas temperature $T_{\mathrm{CO}}(s)$. Following Beckwith \& Sargent (1993, and references therein), the absorbing cross section $\sigma_{v}(s)$ can be expressed in term of the integrated cross section of the transition $\sigma_{0}$ through the relation

$\sigma_{\nu}(s)=\sigma_{0} \cdot \phi_{\nu}(s) \cdot\left(1-\mathrm{e}^{-h v / k T_{\mathrm{CO}}(s)}\right)$,

where $\phi_{v}(s)$ is the intrinsic line profile

$\phi_{\nu}(s)=\frac{c}{v_{0}} \cdot \frac{1}{\Delta V \sqrt{\pi}} \cdot \exp \left(-\frac{\Delta^{2} v}{\Delta^{2} V}\right)$,

with

$\Delta V=\sqrt{\frac{2 k T_{\mathrm{CO}}(s)}{m_{\mathrm{CO}}}+v_{\mathrm{turb}}^{2}}$

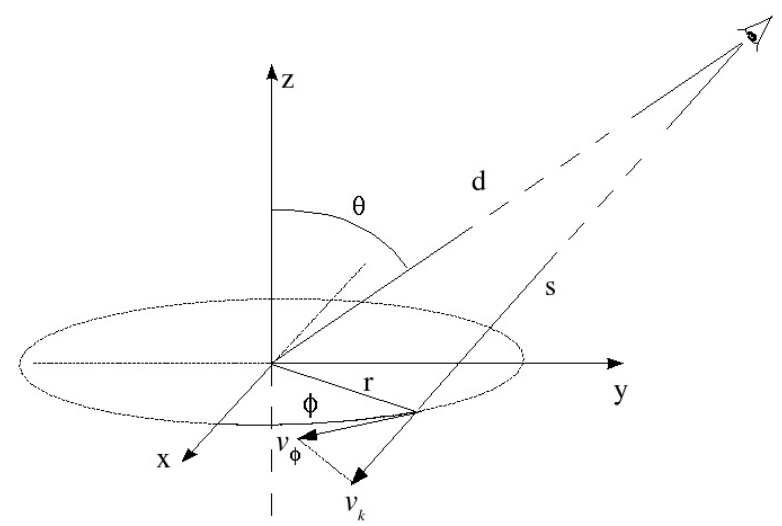

Fig. A.1. Schematic representation of the frame of reference adopted to calculate the $\mathrm{CO}$ emission arising from a rotating disk. The disk midplane and the observer lie respectively on the $(x, y)$ and $(y, z)$ planes; $\theta$ is the disk inclination; $d$ is the distance between the observer and the central star; $s$ is the linear coordinate along the line of sight increasing from the observer $(s \equiv 0)$ towards the emitting source. Assuming that the material within the disk is subject to the Keplerian rotation around the central star, we call $v_{\phi}$ the velocity of a mass element at distance $r$ and $v_{k}$ the projection of $v_{\phi}$ along the line of sight.

and

$\sigma_{0}=\frac{8 \pi^{3} k T_{1}}{h^{2} c} \frac{(l+1)^{2}}{2 l+1} \mu^{2}$.

In the previous equations, $m_{\mathrm{CO}}$ is the $\mathrm{CO}$ molecular weight, $v_{0}$ is the rest frequency of the molecular transition, $\Delta v$ is the difference between the velocity $v_{\mathrm{obs}}=\left(c / v_{0}\right)\left(v-v_{0}\right)$, corresponding to the frequency $v$, and the component of the gas velocity along the line of sight, $v_{k}(s), \mu$ is the dipole moment of the $\mathrm{CO}$ molecule. Note that writing Eq. (A.7), we assumed that the intrinsic line width depends both on the thermal velocity dispersion in the gas and on turbulent velocity $v_{\text {turb }}$.

As shown in Fig. 5 and described in Sect. 3.2, the observed velocity patterns in the $\mathrm{CO}$ transitions are in good agreement with the Keplerian rotation of the disk. We can thus assume that gas moves on circular orbits around the star characterized by a tangential velocity

$v_{\phi}(r)=\sqrt{\frac{\mathrm{G} M_{\star}}{r}}$,

where $r$ is the radius of the orbit and $M_{\star}$ is the stellar mass. To calculate the velocity $v_{k}(s)$, component of the tangential velocity $v_{\phi}(r)$ along the line of sight, it is useful to define a coordinates system centered on the star as shown in Fig. A.1: the $(x, y)$ plane corresponds to the disk midplane; the observer lies in the $(y, z)$ plane and its position it is defined by the inclination $\theta$ and the distance $d$ from the star; each point of the circumstellar space can be defined through the cylindrical coordinates $r=\sqrt{x^{2}+y^{2}}, \phi=\arctan (y / x)$ and $z$. Since $d \gg r$, we can write $v_{k}(s) \cong v_{\phi}(r) \cdot \cos \phi \cdot \sin \theta$.

Finally, the velocity $v_{k}(s)$ can be calculated for each direction, knowing the geometrical transformations between the coordinate $s$ along the line of sight and the cylindrical coordinates $r$ and $\phi$.

In order to solve the described set of equations, we thus need an expression for the circumstellar mass density $\rho(s)$ and the temperature of the emitting gas $T_{\mathrm{CO}}(s)$. In both cases we can 

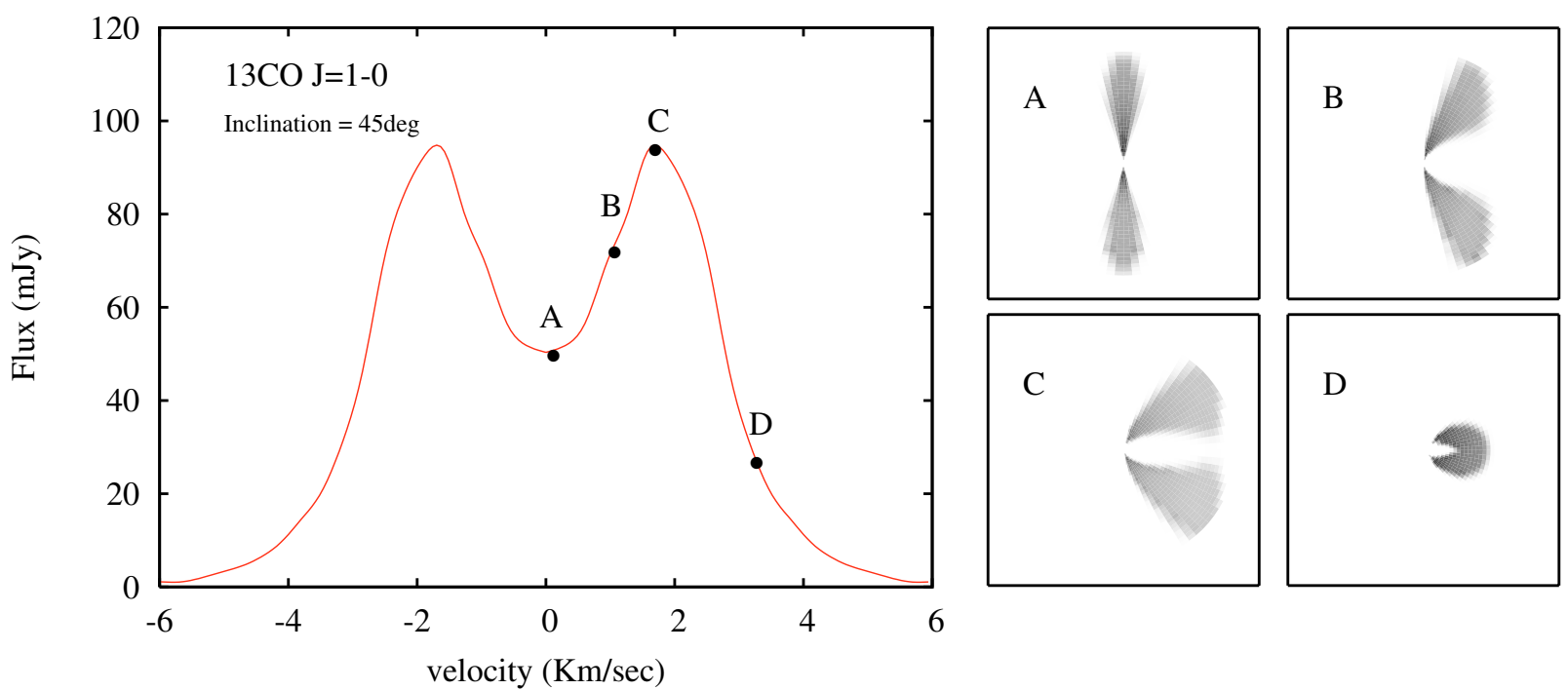

Fig. A.2. ${ }^{13} \mathrm{CO} J=1-0$ simulated emission for a Keplerian disk inclined by $45^{\circ}$ rotating around a central star of $1 M_{\odot}$. The disk outer radius is $R_{\text {out }}=100 \mathrm{AU}$ with a surface density $\Sigma(r) \propto r^{-1.5}$. The left panel show the spatial integrated line profile. The right panel shows the spatially resolved maps at different velocity as labelled in figure.

assume the cylindrical symmetry and write $\rho(s) \equiv \rho(r, z)$ and $T_{\mathrm{CO}}(s) \equiv T_{\mathrm{CO}}(r)$.

For the emitting gas temperature, we choose the parameterization

$T_{\mathrm{CO}}(r)=T_{\mathrm{CO}}\left(r_{0}\right)\left(r / r_{0}\right)^{-q}$,

while the mass density is calculated assuming that the disk is in hydrostatic equilibrium and vertically isothermal in the inner region at the midplane temperature $T_{\mathrm{m}}(r)$, through the relation

$\rho(r, z)=\rho_{0}(r) \cdot \mathrm{e}^{-z^{2} / 2 h^{2}(r)}$,

valid between the disk inner and outer radii $R_{\text {in }}$ and $R_{\text {out }}$. The density on the disk midplane $\rho_{0}(r)$ can by expressed as function of the surface mass density $\Sigma(r)=\Sigma_{0}\left(r / r_{0}\right)^{-p}$ through the relation

$\rho_{0}(r)=\frac{\Sigma(r)}{\sqrt{2 \pi} h(r)}$.
Finally, the pressure scale $h(r)$ is given by the relation

$h(r)=\sqrt{\frac{2 r^{3} k T_{i}(r)}{G M_{\star} m_{0}},}$

where $T_{i}(r)$ is obtained solving the structure of a stellarirradiated passive disk as described in Dullemond et al. (2001).

Note that the emitting gas temperature $T_{\mathrm{CO}}$ has been parametrized independently of the disk interior temperature $T_{i}$, which governs the density structure of the disk. As pointed out by Dartois et al. (2003), the more optically thick CO transitions may by good tracer of the disk surface where the gas temperature is different from the disk interior.

The resulting model, produces brightness maps for each frequency (see Fig. A.2) that can be compared with the observations presented in Figs. 2 and 3. 